\title{
Fast Auto-Align ment Underwater Wireless Optical Communications Employing Orbital Angular Momentum Modes
}

Chengkun Cai ( $\nabla$ chengkuncai@hust.edu.cn)

Huazhong University of Science and Technology https://orcid.org/0000-0003-4009-338X

Yifan Zhao

Wuhan National Laboratory of Optoelectronics

Jieying Zhang

Wuhan National Laboratory of Optoelectronics

Lulu Wang

Wuhan National Laboratory of Optoelectronics

Jian Wang

Wuhan National Laboratory of Optoelectronics

Research

Keywords: Underwater Optical Communication, Orbital Angular Momentum, Auto-alignment system

Posted Date: April 2nd, 2020

DOI: https://doi.org/10.21203/rs.3.rs-19606/v1

License: (9) This work is licensed under a Creative Commons Attribution 4.0 International License.

Read Full License 


\title{
Fast Auto-Alignment Underwater Wireless Optical Communications Employing Orbital Angular Momentum Modes
}

\author{
Cheng-Kun Cai, Yi-Fan Zhao, Jie-Ying Zhang, Lu-Lu Wang, and Jian Wang* \\ Wuhan National Laboratory for Optoelectronics, School of Optical and Electronic Information, \\ Huazhong University of Science and Technology, Wuhan 430074, Hubei, China \\ *Correspondence to: jwang@hust.edu.cn
}

\begin{abstract}
Underwater wireless communication plays an increasingly important role in more and more emerging ocean and sea activities. Traditional underwater acoustic communication is limited by low transmission capacity (tens of Kbit/s). Underwater wireless optical communication (UWOC) provides high bandwidth $(\mathrm{GHz})$. Yet the challenge is not only its continuous capacity scaling, but its resistance to environment disturbance, which is an intrinsic weakness of wireless optical communications with high directivity. Here, we propose and demonstrate fast auto-alignment underwater wireless optical communications employing orbital angular momentum (OAM) modes. The OAM multiplexing, an alternative approach of space-division multiplexing, enables capacity scaling. The fast auto-alignment system, having two stages for thorough alignment, facilitates stable and reliable communication link against vibration. Two OAM modes multiplexing transmission link with 4-Gbit/s aggregate capacity is demonstrated in the experiment under four different vibration conditions assisted by the fast auto-alignment system with a response time of $244 \mathrm{~Hz}$. The demonstrations may open up new perspective to robust stable underwater wireless optical communications exploiting spatial modes in practical environment.
\end{abstract}

Keywords: Underwater Optical Communication, Orbital Angular Momentum, Auto-alignment system

\section{Introduction}

In the last few years, terrestrial, space and underwater wireless optical communications have been widely explored for ever-increasing data capacity demands [1, 2]. Compared with terrestrial and space links, underwater wireless optical links are relatively less explored due to their complexity [3]. Various physical processes in underwater environments make stable underwater wireless optical links hard to realize [4]. However, owing to great commercial, military and scientific value, the interest towards underwater wireless optical communication has increased $[5,6]$. The performance of underwater acoustic communication, which is the mainstream technology for underwater communications, is limited by the shortcomings of low bandwidth, high delay, low energy efficiency, time varying multipath propagation and Doppler spread [7-9]. Underwater wireless optical communication without these drawbacks is a better option, especially for high data rate transfer in short distances. Apropos of the distance limitation due to the severe water absorption at optical frequency, it is fortunate that there is a relatively low attenuation optical window of blue and green, which is capable of proving high bandwidth underwater communication over adequate distance [10-12]. 
The space-division multiplexing (SDM) is a promising technique for increasing the capacity scalability in free space and optical fiber communications [13]. To improve the transmission capacity, the mode-division multiplexing (MDM) with orbital angular momentum (OAM) modes, one subset of SDM, has gained increasing interest [14-19]. Recently, OAM has been introduced into underwater wireless optical communications [20-24] with impressive performance. OAM mode set is an option for MDM for the potentiality to accommodate infinite states with orthogonality. With different OAM modes carrying different data information, the OAM multiplexing technique can improve the transmission capacity of the communication system $[10,25]$.

It is known that an uninterrupted link is very essential in underwater wireless optical communications. It is also a big challenge to maintain such a reliable link because misalignments caused by underwater vehicles, ocean current or other turbulent sources are unavoidable in practical underwater systems [26]. In practice, the high directivity of the optical beam turns to be counterproductive in underwater wireless optical links. The fluctuation of light beam originating from machine vibration is a major elements for loss of signal. To get a steady and anti-interference output, a fast auto-alignment system would be highly desired.

In this paper, we demonstrate a fast auto-alignment OAM-based 2-meter underwater wireless optical link. Two OAM modes with topological charges of +3 and -3 are multiplexed. The intermodal crosstalk after underwater propagation is less than $-9 \mathrm{~dB}$. Data-carrying OAM multiplexing with quadrature phase-shift keying (QPSK) signals is demonstrated under different vibration conditions.

Methods

\section{Methods}

\section{Concept and principle}

The concept and principle of fast auto-alignment underwater wireless optical link employing OAM modes are illustrated in Figure 1. The transmitter side sends optical signals to the receiver side. The MDM technology with OAM modes is employed to increase the transmission capacity. The fluctuation of light beam originating from machine vibration is a major elements for the loss of signal in the underwater transmission link. To overcome the beam fluctuation and get a stable output, the fast auto-alignment system is built.

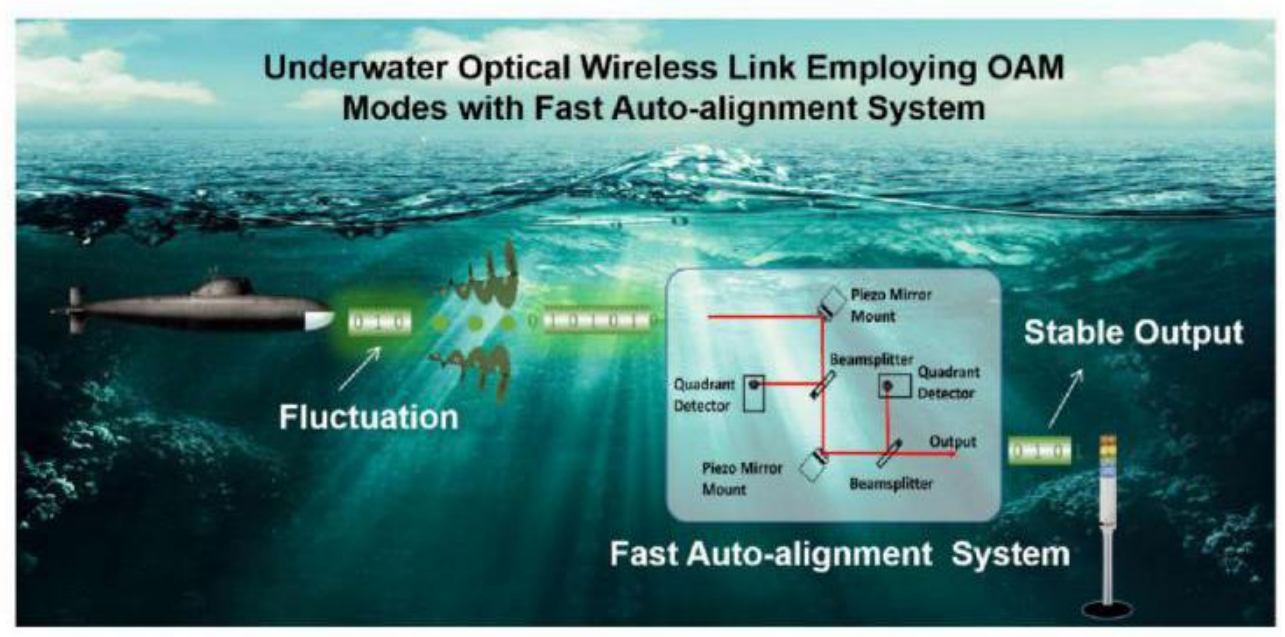

Fig. 1. Concept and principle of fast auto-alignment underwater wireless optical link employing OAM modes. 
The schematic diagram of the fast auto-alignment system is shown in Figure 2(a). The system consists of two alignment stages. Each alignment stage consists of one quadrant detector, one position sensing detector (PSD) auto aligner, two piezo controllers, one beamsplitter, and one piezo mirror mount. The quadrant detector can accurately measure the displacement of an incident beam relative to the calibrated center. If a symmetrical beam is centered on the sensor, four equal photocurrents will be detected, resulting in null difference signals. The photocurrents will change if the beam moves off center, thereby giving rise to difference signals that are related to the beam displacement from the center of the sensor. The quadrant detector is connected with the PSD auto aligner. The PSD auto aligner can read the difference signals (X DIFF and Y DIFF) of the quadrant. The digital signal processor (DSP) inside the PSD auto aligner deals with the difference signals and outputs the corresponding position demand signals which will be used as the inputs to the piezo drivers. When piezo driver is operated together with the PSD auto aligner, high-precision closed-loop operation can be achieved due to the complete feedback. The piezo delivers the drive voltage which is proportional to the position demand signals to drive the piezo mirror mount. The piezo mirror mount is driven by the drive voltage to center the beam on the detector. The beamsplitter sends part of the beam to the quadrant position sensor. The quadrant position sensor can acquire the difference in quantity of the beam' s position relative to the detector center. The computer is used to control and monitor the whole feedback system.

(a)

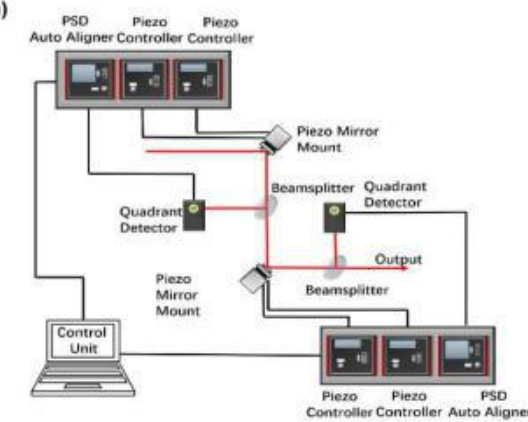

(c)

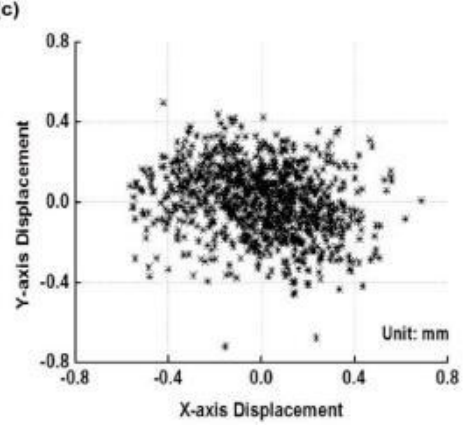

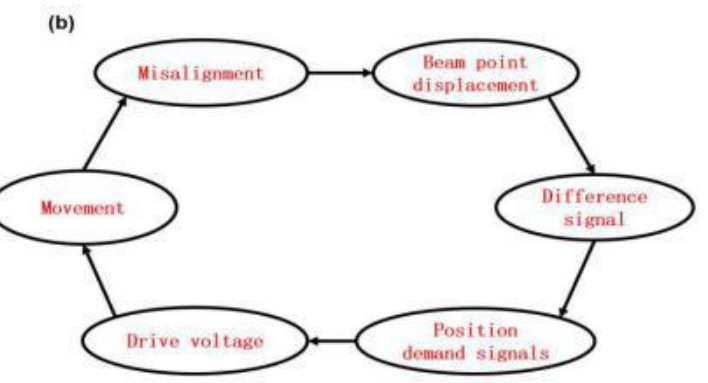

(d)

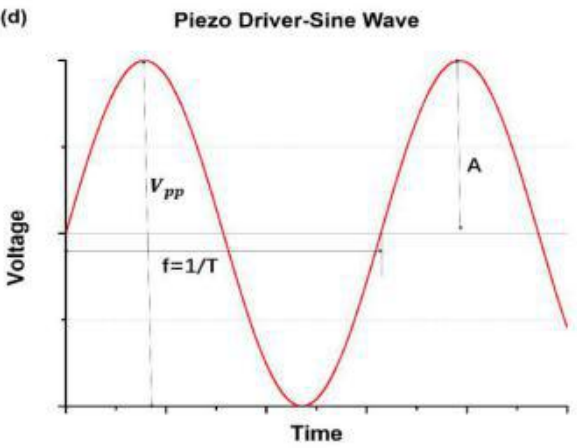

Fig. 2. (a) Schematic diagram of the fast auto-alignment system. (b) Flow chart of auto-alignment process. (c) Scatter plot of beam's displacements. (d) Diagram of drive voltage as a function of the time.

The flow chart of the auto-alignment process is shown in Figure 2(b). The light is incident on the first tuning mirror mount. The tuning mirror directs light to a beamsplitter which divides the light into two parts. The reflected light is directed towards the quadrant detector. The misalignment of the link caused the displacement of the beam point on the quadrant detector which outputs difference signals in horizontal and vertical direction. And the displacements are proportional to the intensity of signals. The PSD auto aligner reads the difference signals and outputs the corresponding position demand signals. 
The piezo drivers receive the position demand signals and output corresponding drive voltages which drive the piezo mirror mount to center the beam on the detector. The detector is sensitive to the beam's power density. By this way, the laser beam can be fixed on the center of the detector array. The transmitted light from the beamsplitter would go through the second alignment stage which is the same as the first alignment stage. So light beams would fixed on the center of quadrant detectors. Two fixed points determine a fixed line. When the two alignment stages are arranged on the proper position, the complete feedback system is able to settle the beam's trajectory in a specific path.

The capability to stabilize the beam, which can be represented by the response time, is a essential feature for a piezo controller in high-speed applications. The response time mainly results from the charging and discharging process of the piezo driver and can be calculated through the process. Figure 2(c) shows a large sample of beam' s displacements in the experiment. According to the flow chart, the beam's displacements, the difference signals, the position demand signals and the drive voltages all are proportional to each other. So each point in Figure 2(c) also represents two drive voltages. The auto-alignment system would center every non-central point. The response time of the system is also the time when the voltage value becomes zero. The movements of the beam are random, which means the average time of the voltage variations is needed. The average drive voltage can be calculated according to the scatter plot, which is about $10 \mathrm{~V}$. The bandwidth would be calculated by the given parameters. The piezo controller can achieve up to $7.5 \mathrm{~mA}$ current. And the load capacitance of the pizeo is $1 \mathrm{uF}$. The response time would be lager if the capacitance is higher. The signal voltage amplitude is determined to be $37.5 \mathrm{~V}$, which represents the length that the piezo extends. The function of a sinusoidal signal with peak amplitude A, average drive voltage Vpp, and frequency $f$ is shown below.

$$
\mathrm{V}(t)=A \sin (2 \pi f t)+A
$$

The Figure 2(d) shows the diagram of the voltage fluctuation. The max response frequency at the steepest slew rate point is reached at $\mathrm{t}=2 \mathrm{npi}(\mathrm{n}=0,1,2, \ldots)$.

$$
\frac{\mathrm{d} V}{d t}=2 \pi A f_{\max }
$$

Thus

$$
f_{\max }=\frac{I_{\max }}{2 \pi A C}=\frac{I_{\max }}{\pi V_{p p} C}
$$

By calculation the response frequency would be about $244 \mathrm{~Hz}$.

\section{Experimental setup}

Figure 3 shows the experiment setup of underwater wireless optical communication with fast auto-alignment system. The arbitrary waveform generator (AWG) generates two electrical 1-Gbaud signals with QPSK modulation format. Two electrical amplifers (EAs) amplifies the signals respectively. And two 520-nm single mode pigtailed laser diodes (LD) modulate the signals directly. The collimators (Col.) are able to couple the Gaussian mode signals from fiber to free space. Two phase-only spatial light modulators (SLMs) convert the Gaussian mode beams into OAM beams by loading complex phase masks. For efficient phase modulation, the polarization states of signals are aligned to the optimal working direction by using the two half-wave plates (HWP). A beam splitter (BS) combines the two spatially orthogonal OAM beams together. To select the desired output and remove 
other unwanted diffraction orders, a pinhole is used as a spatial filter. To reduce the beam size, two lenses work together as an inverse telescope system.

To generate the vibration, the mirror is connected with a motor. A 2-meter-long rectangular tank (40 cm width $\mathrm{x} 40 \mathrm{~cm}$ height) filled with tap water is used to emulate the underwater condition.
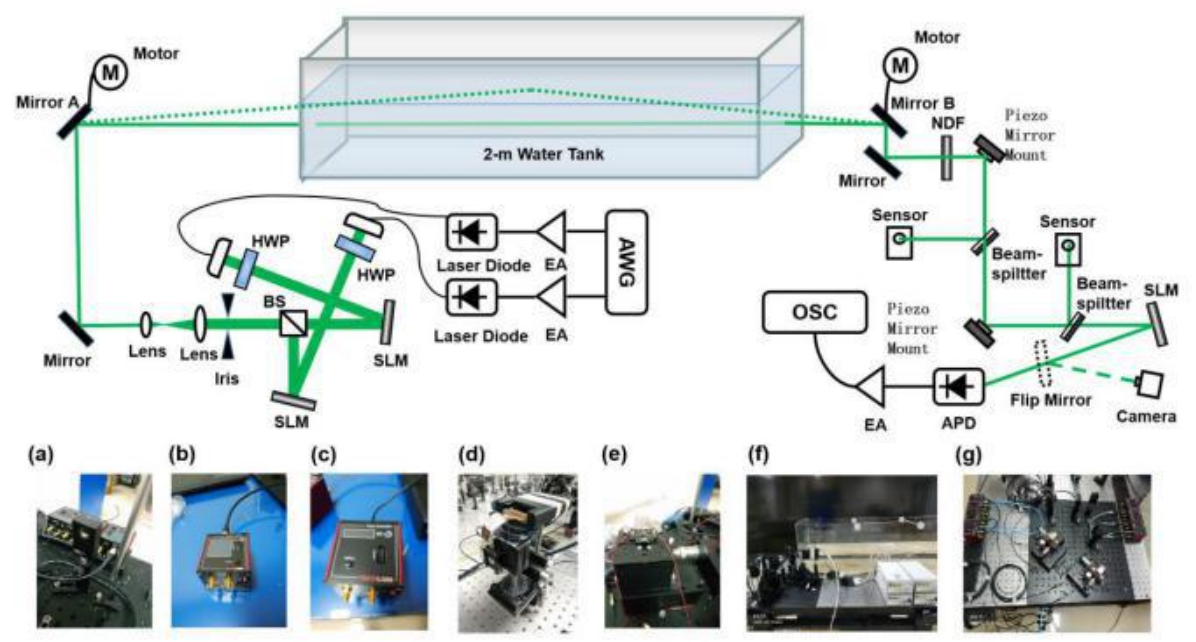

Fig. 3. Experimental setup of underwater wireless optical link employing OAM modes with fast auto-alignment system. (a) Quadrant detector (sensor). (b) PSD auto aligner. (c) Piezo driver. (d) Spatial light modulator. (e) Piezo mirror mount. (f) Water tank, (g) Auto-alignment system.

We connect mirror with motor to simulate the vibration conditions. The experiments are processed under four different conditions: (a) vibration from the transmitter side; (b) vibration from receiver side; (c) vibration from both transmitter side and receiver side; (d) non-line-of-sight link, vibration from both transmitter side and receiver side. The OAM modes multiplexing light propagates through the 2-meter underwater condition. A neutral density filter (NDF) is placed before the fast auto-alignment system to adjust the received optical power.

The received light beams after the fast auto-alignment system are demodulated by the SLM on the received side with a switchable specific fork phase pattern for different OAM channels. After the demodulation, the center of the beam, the intensity profile of which is a bright spot, is sent to a high sensitivity silicon avalanche photodiode detector (APD). The signal detected by the APD are amplified by another electrical amplifier (EA). The amplified signal is received by the oscilloscope (OSC) for bit-error rate (BER) performance measurement. To record the intensity profile of the light beam, a camera assisted by a flip mirror (FM) is used.

\section{Results and Discussion}

To study the multiplexing process of the system, the OAM mode states before and after the transmission are recorded. The left part of figure 4 shows the transmitted side beam intensity profiles with corresponding patterns on the SLMs. At the transmitted side, two different modulated OAM modes with topological charges of +3 and -3 are perfect with doughnut profiles. 


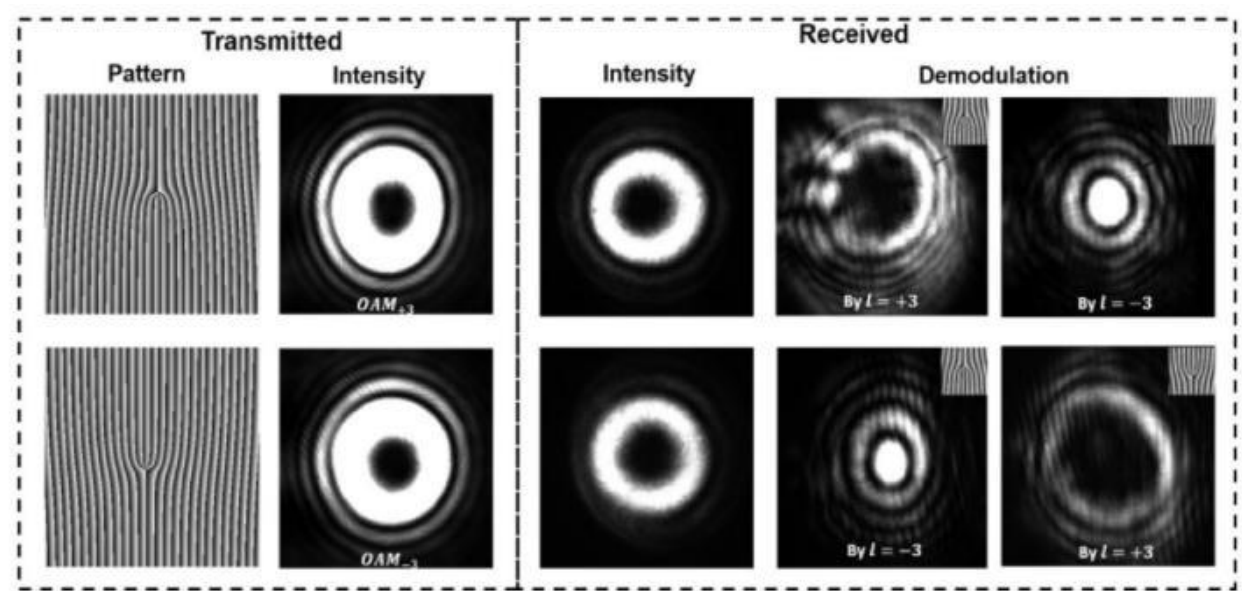

Fig. 4. Measured intensity profiles for and at the transmitter side (Tx), receiver side (Rx) and the side after demodulation by patterns.

After the 2-meter underwater propagation, the intensity profiles of the received light beams still maintain complete with negligible deformation. The intensity profiles of the demodulated light beam have a Gaussian-like bright center spot. When the pattern on the received side is the same as the pattern on the transmitted side, the topological charge of the OAM beam is doubled up to $+6 /-6$, leading to a larger doughnut profile, as shown in Figure 4.

To evaluate the performance of the fast auto-alignment system, we simulate the vibration of underwater working machine by using a mirror with a vibrating motor. When reflected by the oscillatory mirror, the affected light would propagate in a random orientation. 1000 pictures with an time interval of $1 / 30$ second are taken at the Camera- 1 to record the beam trajectory. We make scatter plots of the displacements of the spot which represent the vibration degree of the beam center. The larger the scatter distribution, the more serious the vibration degree is. Figures 5(a)-5(d) show the beam' s trajectory of 1000 point without the fast auto-alignment system under the four conditions: (1) vibration from the transmitter side; (2) vibration from the receiver side; (3) vibration from both transmitter side and receiver side; (4) non-line-of-sight link, vibration from both transmitter side and receiver side. Figures 5(e)-5(h) show the beam's trajectory of 1000 point with the fast auto-alignment system under the four conditions. Under the condition (1) in Figures 5(a) and 5(e), the maximum variation of scatter distribution shrinks from $0.7 \mathrm{~mm}$ to about $0.2 \mathrm{~mm}$ after the fast auto-alignment process.

Under the condition (2) in Figures 5(b) and 5(f), the maximum variation of scatter distribution shrinks from $0.7 \mathrm{~mm}$ to about $0.15 \mathrm{~mm}$ after the fast auto-alignment process. Under the condition (3) in Figures $5(\mathrm{c})$ and $5(\mathrm{~g})$, the maximum variation of scatter distribution shrinks from $0.6 \mathrm{~mm}$ to about 0.2 $\mathrm{mm}$ after the fast auto-alignment process. Under the condition (4) in Figures 5(d) and 5(h), the maximum variation of scatter distribution shrinks from $0.7 \mathrm{~mm}$ to about $0.2 \mathrm{~mm}$ after the fast auto-alignment process. One can clearly see that after the auto-alignment process, the distribution range of the beam is obviously reduced, which represents the excellent performance to stabilize the light path. 


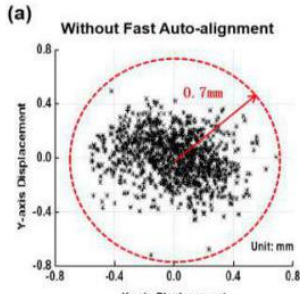

(e)

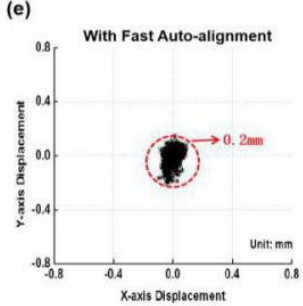

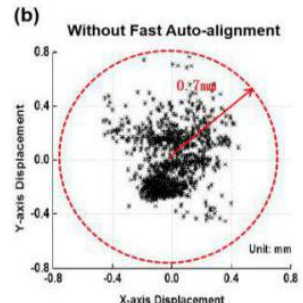

(f)

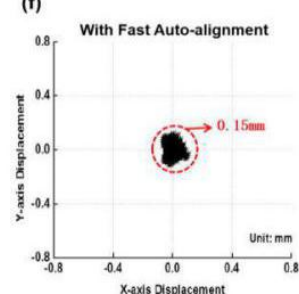

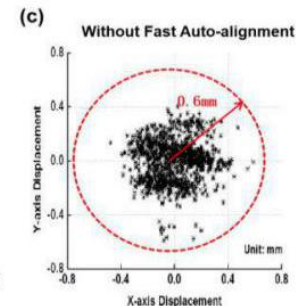

(g)

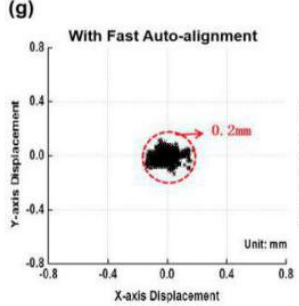

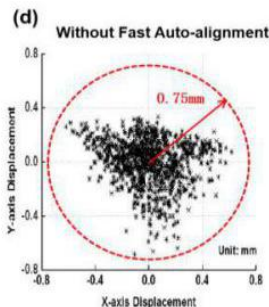

(h)

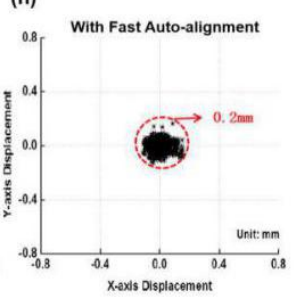

Fig. 5. Beam's trajectory under different vibration condition. (a)-(d) Without fast auto-alignment. (e)-(h) With fast auto-alignment. (a)(e) Vibration from the transmitter side. (b)(f) Vibration from the receiver side. (c)(g) Vibration from both transmitter side and receiver side. (d)(h) Non-line-of-sight link, vibration from both transmitter side and receiver side.
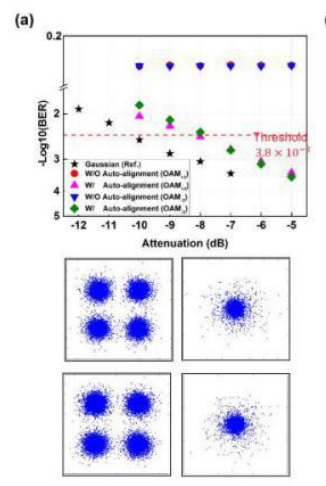
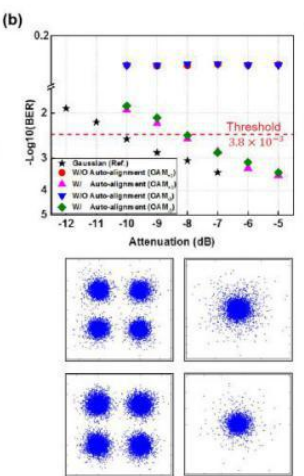
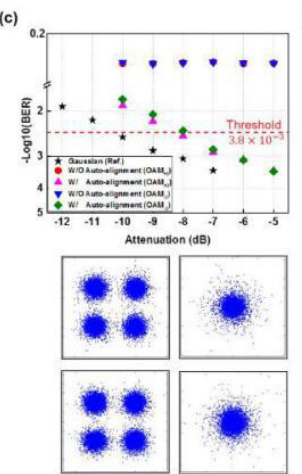
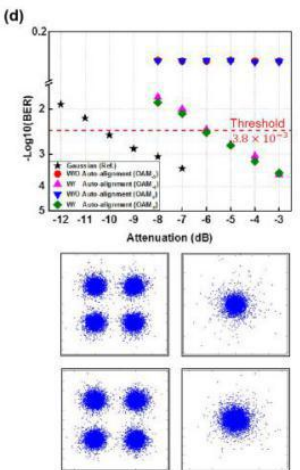

Fig. 6. Measured BER performance with (w/) and without (w/o) fast auto-alignment under different vibration conditions. (a) Vibration from the transmitter side. (b) Vibration from the receiver side. (c) Vibration from both transmitter side and receiver side. (d) Non-line-of-sight link, vibration from both transmitter side and receiver side. The bottom shows the constellation diagrams. Left: w/ fast auto-alignment (re-established link). Right: w/o fast auto-alignment (disconnected link).

The data-carrying performance of the 2-meter underwater OAM modes multiplexing communication is further measured. Each OAM mode carries a 1-Gbaud QPSK signal, thus the aggregate capacity is $4 \mathrm{Gbit} / \mathrm{s}$. When the received powers are the same, the BER values of different OAM modes will be equal. For the power sensitivity of the APD, the attenuation of NDF is a good approach for the BER measurement. Figures 6(a)-6(d) plot the measured BER performance with and without fast auto-alignment under different vibration conditions. The forward error correction (FEC) threshold is $3.8 \times 10-3$. The Gaussian mode is taken as the reference channel. The BER performances of and have no significant difference. From the obtained results, we can see that the optical link is completely disconnect without using the fast auto-alignment system. When using the fast auto-alignment system, the UWOC employing OAM mode multiplexing is re-established and vibration-resistant. With future improvement, more OAM modes and faster auto-alignment system could be considered to further facilitate the capacity scaling and enhance the robustness. The demonstrations pave a way to implement practical underwater wireless optical communications with 
increased transmission capacity by OAM multiplexing and with robustness against environmental disturbance by fast auto-alignment system.

\title{
Conclusions
}

In summary, we have proposed and demonstrated an fast auto-alignment underwater wireless optical system. From the obtained results, we can see that the optical link is completely disconnect without using the fast auto-alignment system. When using the fast auto-alignment system, the UWOC employing OAM mode multiplexing is re-established and vibration-resistant. With future improvement, more OAM modes and faster auto-alignment system could be considered to further facilitate the capacity scaling and enhance the robustness. The demonstrations pave a way to implement practical underwater wireless optical communications with increased transmission capacity by OAM multiplexing and with robustness against environmental disturbance by fast auto-alignment system.

\begin{abstract}
Abbreviations
UWOC:Underwater wireless optical communication; OAM:orbital angular momentum; SDM:space-division multiplexing; MDM:mode-division multiplexing; QPSK:quadrature phase-shift keying; PSD:position sensing detector; DSP:digital signal processor; AWG: arbitrary waveform generator; EA:electrical amplifers; LD:laser diodes; Col:collimator; SLM: spatial light modulators; HWP:half-wave plates; BS:beam splitter; NDF:neutral density filter; APD:avalanche photodiode detector; OSC:oscilloscope; BER:bit-error rate; FM:flip mirror; FEC:forward error correction.
\end{abstract}

\section{Acknowledgments}

This research was supported by the National Natural Science Foundation of China (NSFC) $(61761130082,11574001,11774116)$; National Key R\&D Program of China (2018YFB1801803); National Program for Support of Top-notch Young Professionals; Natural Science Foundation of Hubei Province of China (2018CFA048); Royal Society-Newton Advanced Fellowship; Key R\&D Program of Guangdong Province (2018B030325002); Open Fund of IPOC (BUPT) (IPOC2018A002); Yangtze River Excellent Young Scholars Program; Program for HUST Academic Frontier Youth Team (2016QYTD05); Fundamental Research Funds for the Central Universities (2019kfyRCPY037).

\section{Authors' contributions}

JW developed the concept and conceived the experiment. C-KC. constructed the experiment. C-KC performed the theoretical analyses. C-KC and J-YZ acquired the experimental data and carried out the data analyses. Y-FZ, J-YZ, L-LW, and JW provided technical supports. All authors contributed to writing. JW finalized the paper. JW supervised the project. All authors read and approved the final manuscript.

\section{Funding}

This work was supported by the National Natural Science Foundation of China (NSFC) $(61761130082,11574001,11774116)$; National Key R\&D Program of China (2018YFB1801803); National Program for Support of Top-notch Young Professionals; Natural Science Foundation of Hubei Province of China (2018CFA048); Royal Society-Newton Advanced Fellowship; Key R\&D Program of Guangdong Province (2018B030325002); Open Fund of IPOC (BUPT) (IPOC2018A002); Yangtze River 
Excellent Young Scholars Program; Program for HUST Academic Frontier Youth Team (2016QYTD05); Fundamental Research Funds for the Central Universities (2019kfyRCPY037).

\section{Availability of data and materials}

The data that support the findings of this study are available from the corresponding author on request.

\section{Competing interests}

The authors declare that they have no competing interests.

\section{Ethics approval and consent to participate}

Not applicable.

\section{Consent for publication}

Not applicable.

\section{References}

1. Stojanovic M, et al. Underwater acoustic communication channels-Propagation models and statistical characterization. IEEE Commun. Mag. 2009;47:0-89.

2. Leonard NE, et al. Collective Motion, Sensor Networks, and Ocean Sampling. Proc. IEEE. 2007;95:48-74.

3. William C, et al. Simulating channel losses in an underwater optical communication system. Opt. Soc. Am. A. 2014;31: 920-934.

4. Kaushal H, et al. Underwater Optical Wireless Communication. IEEE Access. 2016;4:1518-1547.

5. Embley RW, et al. Long-term eruptive activity at a submarine arc volcano. Nature. 2006;441:494-497.

6. Oubei HM, et al. $2.3 \mathrm{Gbit}$ s underwater wireless optical communications using directly modulated $520 \mathrm{~nm}$ laser diode. Opt. Express. 2015;23:20743-20748.

7. Hale GM, et al. Optical Constants of Water in the 200-nm to 200- $\mu \mathrm{m}$ Wavelength Region. Appl. Opt. 1973;12:555-563 .

8. Freitag L, et al. Analysis of channel effects on direct-sequence and frequency-hopped spread-spectrum acoustic communication. IEEE J. Oceanic Eng. 2001;26:586-593.

9. Sharifzadeh M, et al. Performance analysis of underwater wireless optical communication systems over a wide range of optical turbulence. Opt. Commun. 2018;427:609-616.

10. Ren Y, et al. Orbital Angular Momentum-based Space Division Multiplexing for High-capacity Underwater Optical Communications. Sci. Rep. 2016;6:33306.

11. Xu J, et al. Underwater wireless optical communication using a blue-light leaky feeder. Opt. Commun. 2017;397:51-54.

12. Oubei HM, et al. 4.8 Gbit s 16-QAM-OFDM transmission based on compact 450-nm laser for underwater wireless optical communication. Opt. Express. 2015;23:23302-23309.

13. Richardson DJ, et al. Space-division multiplexing in optical fibres. Nature Photon. 2013;7: 354.

14. Wang J. Advances in communications using optical vortices. Photon. Res. 2016;4:B14-B28.

15. Wang J. Data information transfer using complex optical fields: a review and perspective. Chin. Opt. Lett. 2017;15:16-20 .

16. Wang J. Metasurfaces enabling structured light manipulation: advances and perspectives. Chin. Opt. Lett. 2018;16:25-30.

17. Zhu L, et al. $18 \mathrm{~km}$ low-crosstalk OAM+WDM transmission with 224 individual channels enabled by a ring-core fiber with large high-order mode group separation. Opt. Lett. 2018;43:1890-1893.

18. Zhou N, et al. Ultra-compact broadband polarization diversity orbital angular momentum generator with $3.6 \times 3.6 \mu \mathrm{m} 2$ footprint. Sci. Adv. 2019;5:eaau9593.

19. Wang J, Twisted optical communications using orbital angular momentum. Sci. China Phys. Mech. Astron. 2019;62:5-25. 
20. Zhao Y-F, et al. Performance evaluation of underwater optical communications using spatial modes subjected to bubbles and obstructions. Opt. Lett. 2017;42:4699-4702.

21. Zhao Y-F, et al. Demonstration of data-carrying orbital angular momentum-based underwater wireless optical multicasting link. Opt. Express. 2017;25:28743-28751.

22. Wang A-D, et al. Adaptive water-air-water data information transfer using orbital angular momentum. Opt. Express. 2018;26:8669-8678.

23. Zhao Y-F, et al. Feedback-enabled adaptive underwater twisted light transmission link utilizing the reflection at the air-water interface. Opt. Express. 2018;26:16102-16112.

24. Cai C-K, et al. Experimental Demonstration of an Underwater Wireless Optical Link Employing Orbital Angular Momentum (OAM) Modes with Fast Auto-Alignment System. in Optical Fiber Communication Conference (OFC) 2019, paper M3I.4.

25. Baghdady J, et al. Multi-gigabit/s underwater optical communication link using orbital angular momentum multiplexing. Opt. Express. 2016;24:9794 - 9805.

26. Tang S, et al. On Link Misalignment for Underwater Wireless Optical Communications. IEEE Commun. Lett. 2012;16: $1688-1690$ 


\section{Figures}

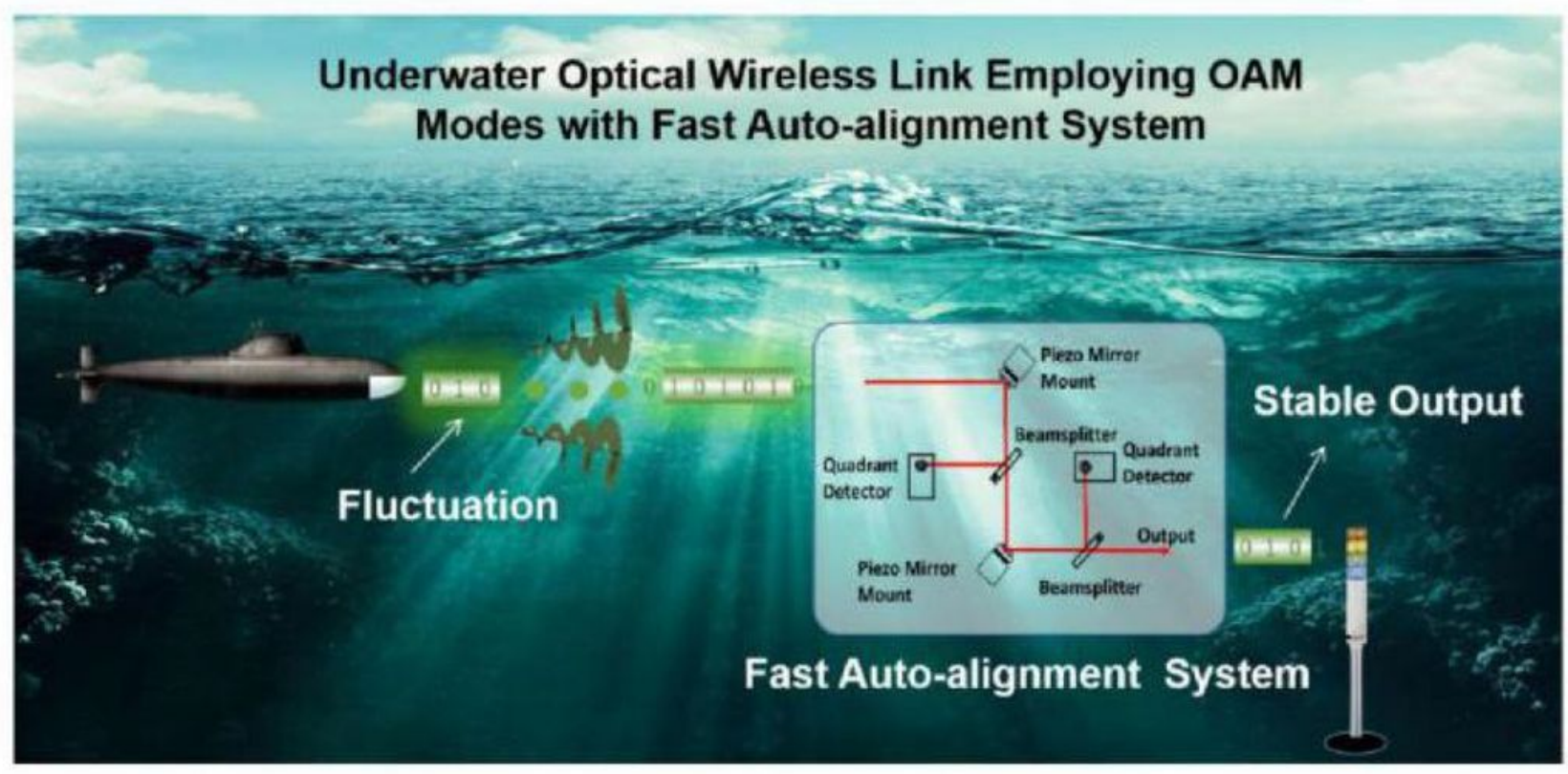

Figure 1

Concept and principle of fast auto-alignment underwater wireless optical link employing OAM modes.

(a)

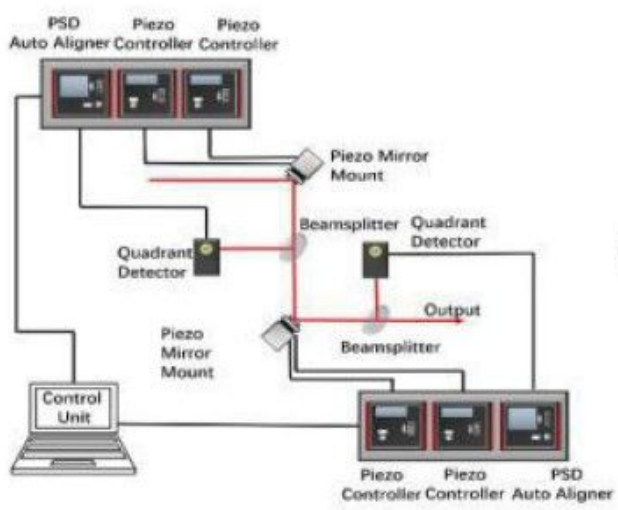

(c)

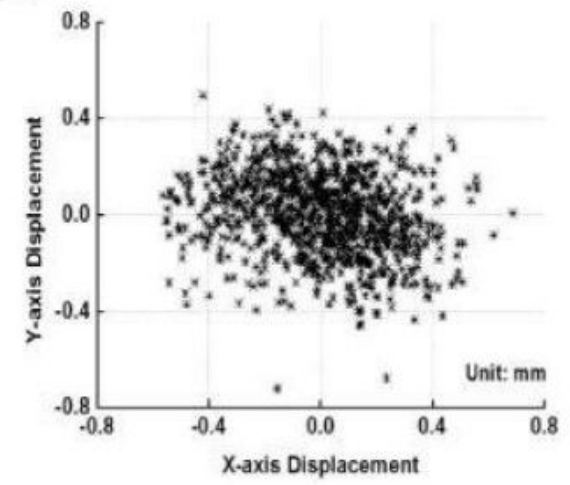

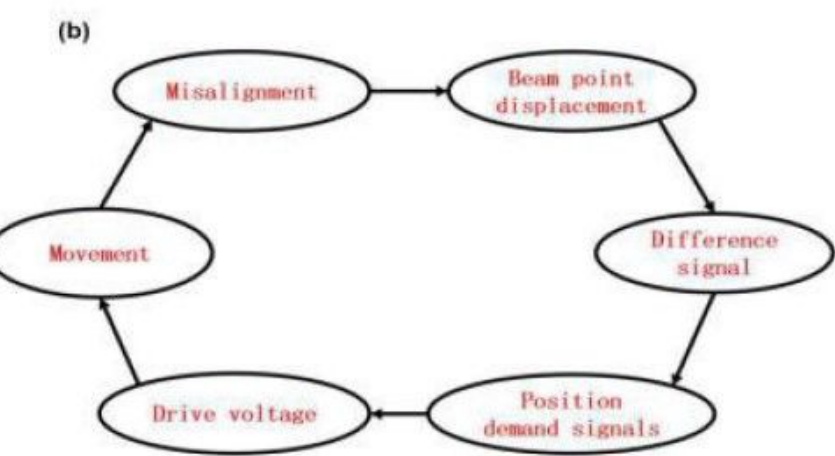

(d)

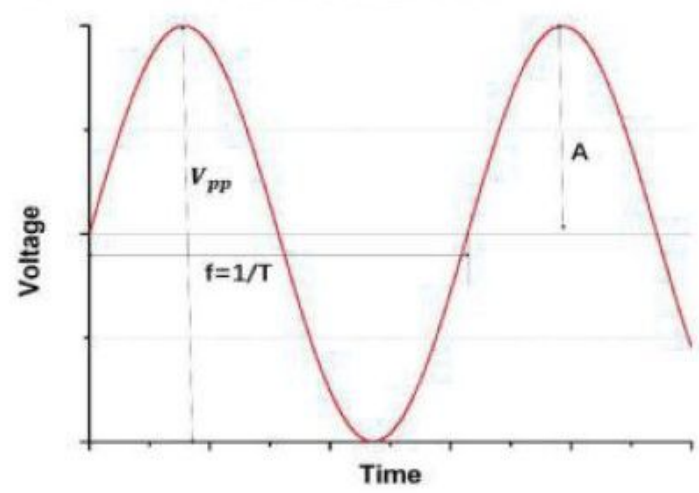


(a) Schematic diagram of the fast auto-alignment system. (b) Flow chart of auto-alignment process. (c) Scatter plot of beam's displacements. (d) Diagram of drive voltage as a function of the time.

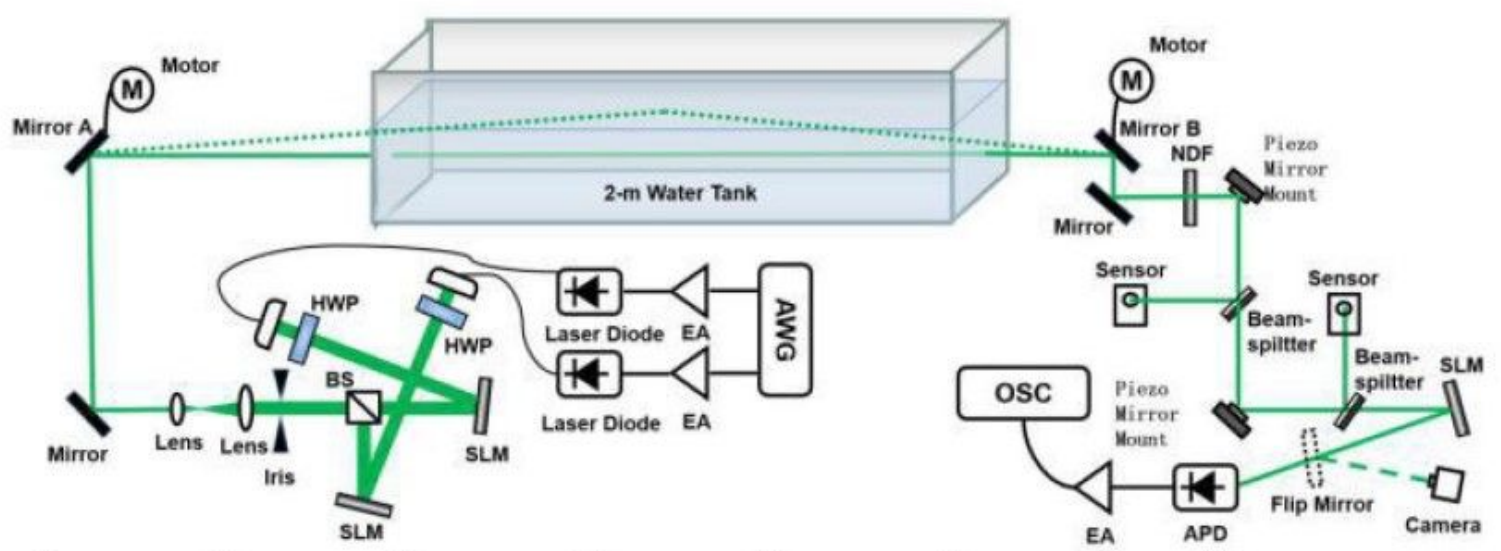

(a)

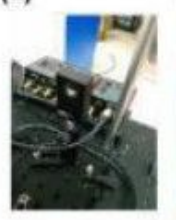

(b)

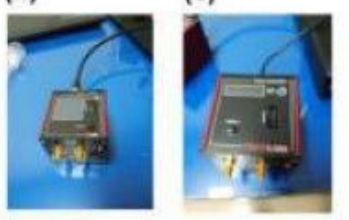

(d)

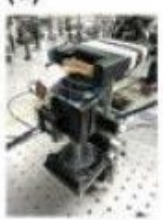

(e)

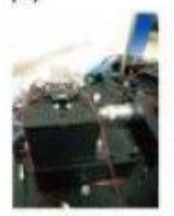

(f)

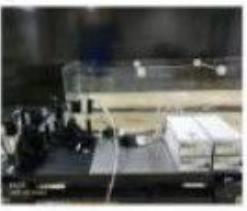

(g)

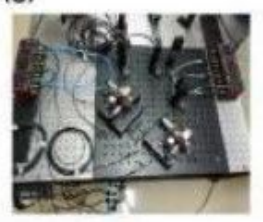

\section{Figure 3}

Experimental setup of underwater wireless optical link employing OAM modes with fast auto-alignment system. (a) Quadrant detector (sensor). (b) PSD auto aligner. (c) Piezo driver. (d) Spatial light modulator. (e) Piezo mirror mount. (f) Water tank, (g) Auto-alignment system.

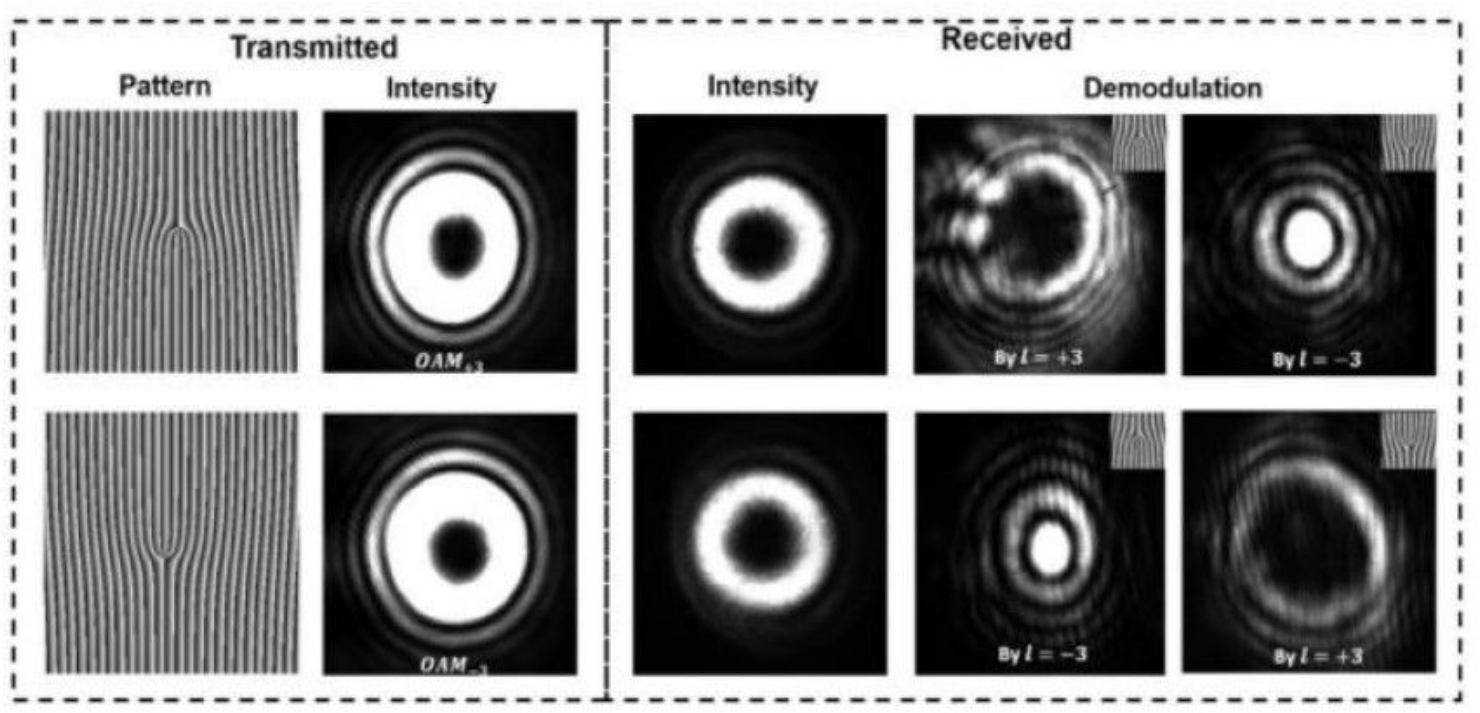

Figure 4 
Measured intensity profiles for and at the transmitter side (Tx), receiver side ( $R x)$ and the side after demodulation by patterns.

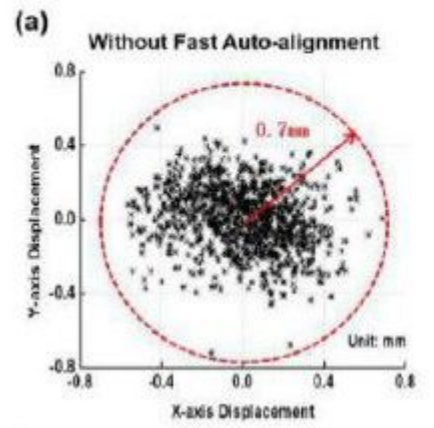

(e)

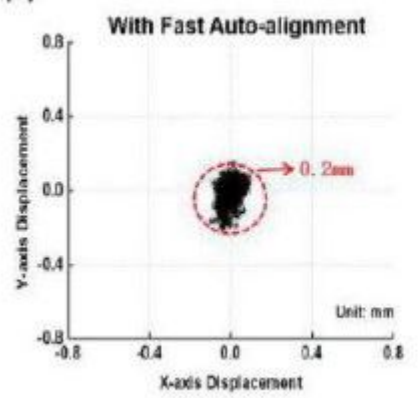

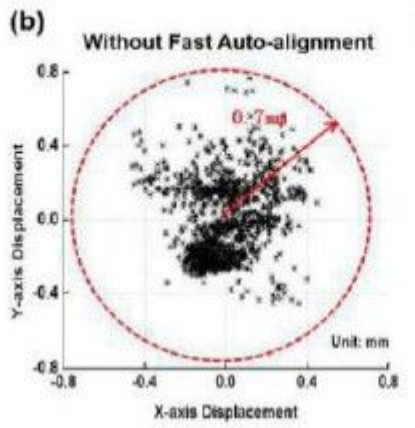

(f)

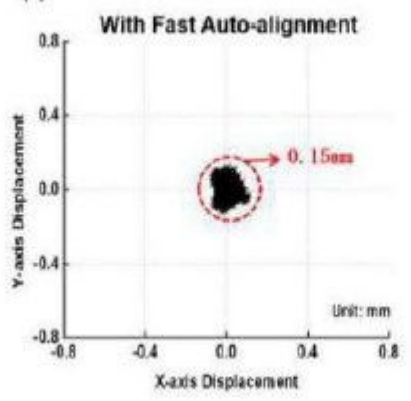

(c)

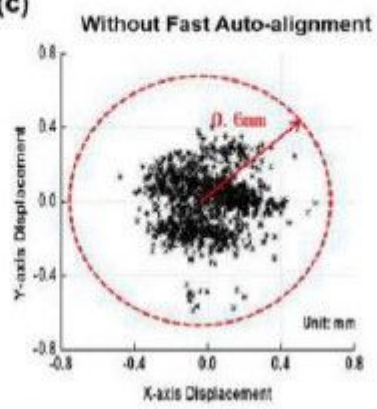

(g)

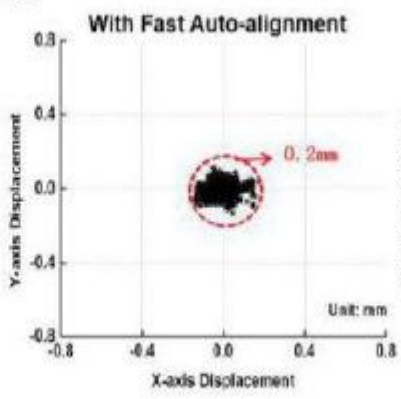

(d) Without Fast Auto-alignment

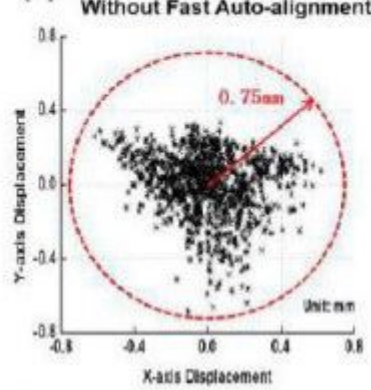

(h)

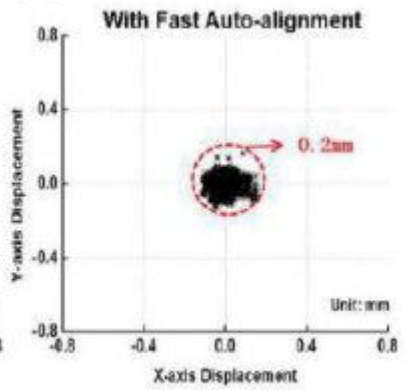

Figure 5

Beam's trajectory under different vibration condition. (a)-(d) Without fast auto-alignment. (e)-(h) With fast auto-alignment. (a)(e) Vibration from the transmitter side. (b)(f) Vibration from the receiver side. (c)(g) Vibration from both transmitter side and receiver side. (d)(h) Non-line-of-sight link, vibration from both transmitter side and receiver side.

(a)
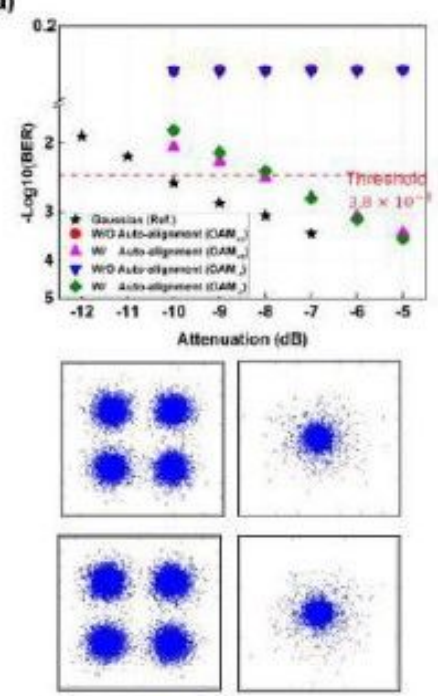

(b)
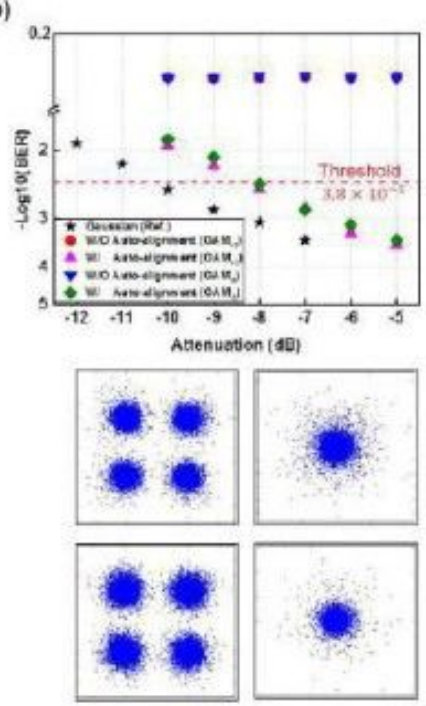

(c)
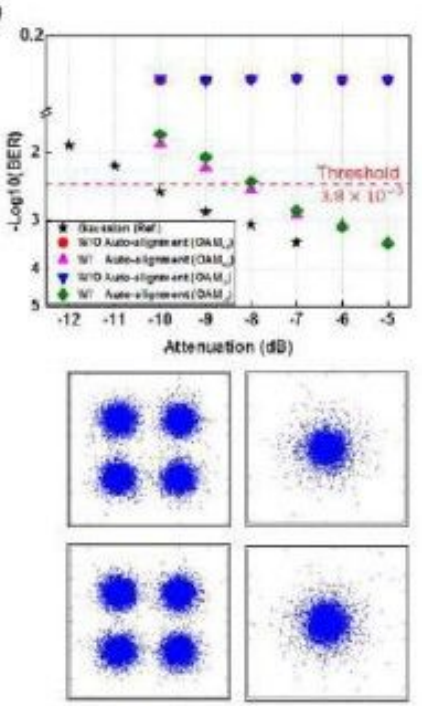

(d)

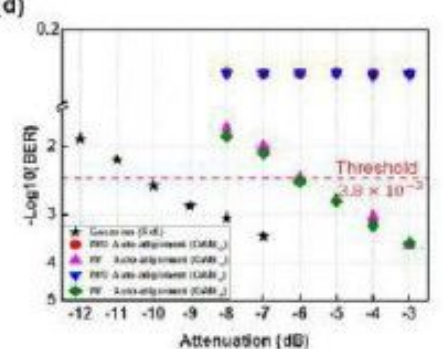

Attrnuasion (dG)
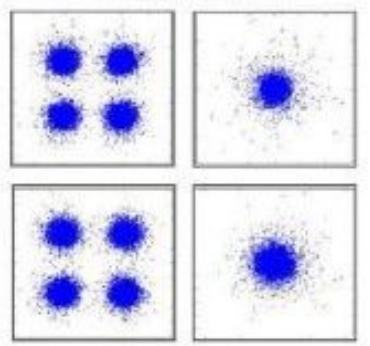

Figure 6 
Measured BER performance with (w/) and without (w/o) fast auto-alignment under different vibration conditions. (a) Vibration from the transmitter side. (b) Vibration from the receiver side. (c) Vibration from both transmitter side and receiver side. (d) Non-line-of-sight link, vibration from both transmitter side and receiver side. The bottom shows the constellation diagrams. Left: $w /$ fast auto-alignment (re-established link). Right: w/o fast auto-alignment (disconnected link). 